\title{
SHOCK-WAVE PROPERTIES OF BRITTLE SOLIDS
}

\author{
D. E. Grady \\ Sandia National Laboratories, Albuquerque, New Mexico 87185-0821
}

\begin{abstract}
Extensive experimental investigation in the form of large-amplitude, nonlinear wave-profile measurements which manifest the shock strength and equation-of-state properties of brittle solids has been performed. Brittle materials for which a base of dynamic property data is available include $\mathrm{Al}_{2} \mathrm{O}_{3}$, $\mathrm{AlN}, \mathrm{B}_{4} \mathrm{C}, \mathrm{CaCO}_{3}, \mathrm{SiC}, \mathrm{Si}_{3} \mathrm{~N}_{4}, \mathrm{SiO}_{2}$ (quartz and glass), $\mathrm{TiB}_{2}$, WC and $\mathrm{ZrO}_{2}$. Planar impact methods and velocity interferometry diagnostics have been used exclusively to provide the high-resolution shock-profile data. These wave-profile data are providing engineering dynamic strength and equationof-state properties as well as controlled, shock-induced motion histories for the validation of theoretical and computational models. Of equal importance, such data are providing a window into the physics of a newly emerging understanding of the compression and deformation behavior of high-strength brittle solids. When considered along with a rich assortment of strength and deformation data in the literature, a systematic assessment of this shock-wave data lends strong support for failure waves and concomitant high-confinement dilatancy as a general mechanism of inelastic deformation in the shock compression of ceramics. Phase transformation in selected brittle solids appears to be a critical state phenomenon strongly controlled by kinetics. The risetime and structure of deformation shock waves in brittle solids are controlled by viscous effects which at present are still poorly understood. The shockwave data also suggest that both crystalline plasticity and brittle fracture may play important and interconnected roles in the dynamic failure process.
\end{abstract}

\section{INTRODUCTION}

Underlying every shock wave event is a shock wave profile which describes the temporal history of the mechanical and thermal state at a material point. In most cases there is not the wherewithal to measure that wave profile. It does, nevertheless, exist and is an issue to consider in the physics of the event.

Over the past three decades a substantial effort in the shock wave community has been focused on the development of diagnostic methods for measuring wave profiles in solids. Numerous physical effects have been exploited in providing a visualization of the wave profile. Piezoresistivity, electroinduction and optical interference are but several of the more successful which continue in active use today.

The discipline of measuring such waves has evolved over the past few decades into a reasonably mature technology with capabilities of measuring waves of stress and particle velocity by several techniques with quite high resolution. This technology was driven by the expectation that such measurements would provide crucial information concerning the transient or high-strain-rate equationof-state and rheological properties of condensed matter.

This expectation has only been realized in part, and it is now well recognized that wave-profile data alone are not sufficient to establish the wide range of material response behavior sought. Such data are only part of a wider range of data from other test methods which are necessary to achieve the desired material response understanding.

This should not in any way diminish the significance of the contributions of the many technical and scientific workers in achieving today's capabilities in the measurement of wave profiles.

There is, perhaps, a higher reason for making such measurements. Wave profiles, like the setting sun, or the splash of a rain drop, are an interesting

This work was suponted by the Uniled

States Depariment of Energy under

Contrict DE-ACD4-94ALP5ONO.

DISTRIBUTION OF THUS DOCUMENT IS UMLMTED TR

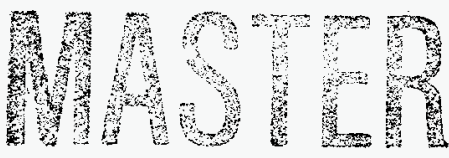




\section{DISCLAIMER}

This report was prepared as an account of work sponsored by an agency of the United States Government. Neither the United States Government nor any agency thereof, nor any of their employees, makes any warranty, express or implied, or assumes any legal liability or responsibility for the accuracy, completeness, or usefulness of any information, apparatus, product, or process disclosed, or represents that its use would not infringe privately owned rights. Reference herein to any specific commercial product, process, or service by trade name, trademark, manufacturer, or otherwise does not necessarily constitute or imply its endorsement, recommendation, or favoring by the United States Government or any agency thereof. The views and opinions of authors expressed herein do not necessarily state or reflect those of the United States Government or any agency thereof. 


\section{DISCLAIMER}

Portions of this document may be illegible in electronic image products. Images are produced from the best available original document. 
phenomenon in the natural world. They are rich in features which stimulate the curiosity toward the underlying responsible physics. Thus, like the sun and the rain, wave profiles in condensed matter deserve, in and of themselves, a satisfactory scientific explanation.

It is for these reasons that the shock wave profile has come to personify the field of shock wave physics.

In this spirit the theme of the present report will focus on shock wave profiles in ceramic materials, or, more generally, wave profiles in that class of materials which we tend to think of as hard and brittle (brittle solids). The experimental method pursued in this investigation has been that of measuring the form and evolution of large amplitude deformation waves produced by carefully controlled impact experiments. The principal diagnostic has been that of velocity interferometry used to measure a time history of velocity at an interface between the solid material of interest and a transparent window material.

Wave profile measurements on brittle solids have revealed an unusually rich display of nonlinear features which hint at the complex interplay of physical processes active during passage of the transient shock wave. Complex failure, rheological and phase transformation processes in solid matter have been put forth to explain such features. The purpose of the present report is to discuss some of the nonlinear features observed in the wave profiles of brittle solids and the possible underlying physics.

\section{PHASE TRANSFORMATION IN THE SHOCK COMPRESSION PROCESS}

The phase transformations expected when lattice instabilities are encountered within the range of pressure excursions experienced in shock wave testing appear to occur with remarkable ease during the brief shock compression process. In many materials transformation kinetics are apparently minimal. In others there are indications that overpressures consistent with the limit of absolute mechanical instability are achieved before transformation is observed. The effects on the evolution of shock profile structure can be remarkable. Wave profile signatures have been observed in a number of the brittle solids examined by the present author and others. Phase transformations in a wide range of materials under shock wave loading have previously been reviewed ${ }^{1,2}$.

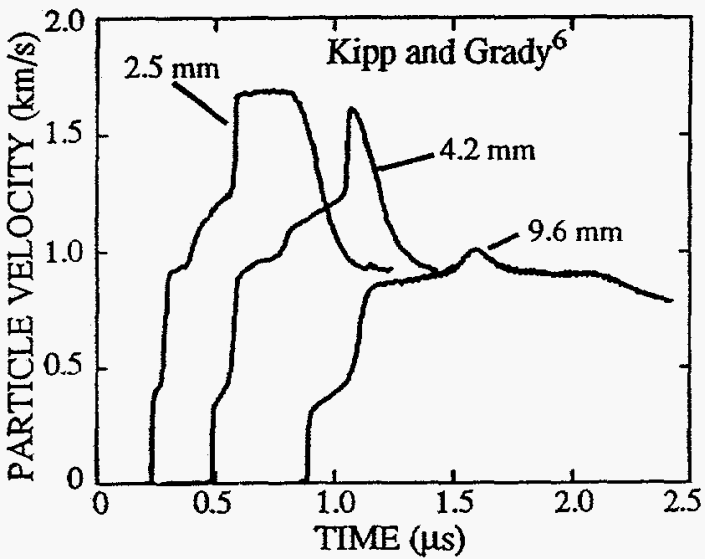

FIGURE 1. Velocity interferometry wave profiles measured in aluminum nitride seramic. Three samples of different thickness were subjected to the same impact velocity. (Ref. 6)

Shock wave data for aluminum nitride ceramic shown in Figure 1 illustrate the extent to which phase transformation can contribute to the complexity of nonlinear wave profiles. In the present experiments shock wave loading to approximately $35 \mathrm{GPa}$ substantially exceeded both the HEL ( $\sim 8 \mathrm{GPa})$ and the wurtzite-to-rocksalt phase transformation stress (20$22 \mathrm{GPa}$ ) in this material. The $20 \%$ volume strain and large dissipation associated with the shock transition process leads to a substantial compression wave splitting and rapid attenuation of the peak wave amplitude. This transformation was first discovered by shock wave techniques ${ }^{3}$ and was subsequently observed through static compression methods. ${ }^{4}$ The static transition pressure of $16.5 \mathrm{GPa}$ reported by Volstadt et al. ${ }^{4}$ would appear to be in remarkably good agreement with the shock transition if elementary corrections for the strength at the shock state are accounted for. ${ }^{5}$ Subsequent shock wave data on aluminum nitride from other suppliers, and with alternative shock diagnostic techniques, have been remarkably consistent in identifying onset of the transition in the 19-22 GPa range. 67

Dandekar et al. ${ }^{8}$ have noted, however, that more recent static studies of the wurtzite-to-rocksalt transition in aluminum nitride seriously clouds this. comparison and that even the issue of material strength in the shock siate leading to the transition 


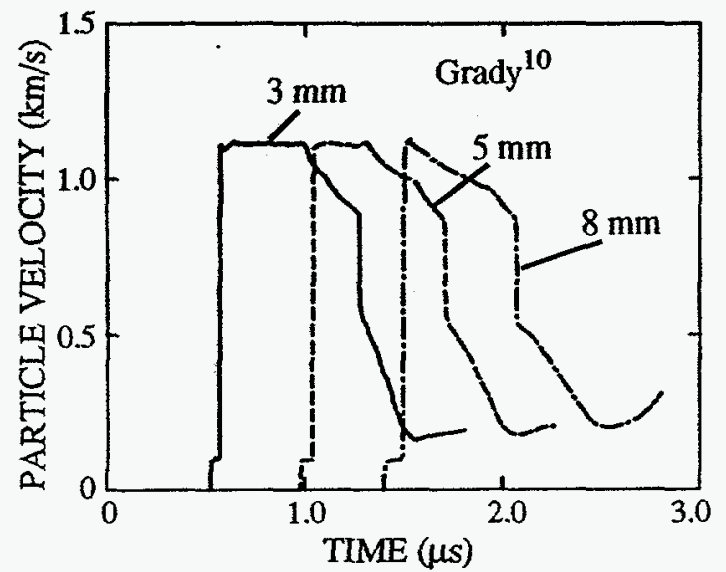

FIGURE 2. Velocity interferometry wave profiles in crystalline calcite. (Ref. 10)

remains an open question. Static transition pressures ranging over 10-23 GPa have now been reported for the aluminum nitride transition by various authors. ${ }^{8}$ Xia et al. ${ }^{9}$ suggest that the equilibrium transition pressure may even be less than $10 \mathrm{GPa}$, and that the wide range of reported values is a consequence of kinetics associated with the reconstructive phase change. That the shock transition proceeds with considerable ease at a stress level near the upper end of the reported static value may simply reflect an overstress sufficient to drive the kinetics, combined with extensive defect production in the shock deformation process. Alternatively, stress levels in the shock state may have achieved the limit of absolute stability (spinodal) for the wurtzite structure.

Unlike aluminum nitride, certain materials have compression shock-wave structures which are silent to the occurrence of phase transformation during the shock compression process. This lack of compressive wave-profile signature can be due both to features of the phase transformation and to material or equationof-state properties leading up to the transformation state. Such behavior is aptly illustrated by the characteristics of the shock-induced phase transformation in calcium carbonate or calcite $\left(\mathrm{CaCO}_{3}\right)$ which occurs under shock compression in a pressure range of approximately $12-15 \mathrm{GPa}^{10}$

Wave profiles measured in crystalline calcite to a maximum shock stress of about $18 \mathrm{GPa}$ are shown in Figure 2. The compressive shock profile shows no indication of transformation within the 12-15 GPa range. The precursor at the substantially lower 1.5-
2.0 GPa level is a consequence of yield and the metastable calcite I-II and calcite II-III transformations. ${ }^{11}$ The lack of a compressive shock wave signature for the higher pressure transformation is due to the relatively small volume change associated with this transition (3-5\%) and the lower pressure calcite I-II-III transitions, with a collective volume strain of about $5 \%$ which effectively centers the shock wave from a slightly distended state. The consequence is that the deformation shock path to the Hugoniot state (the Rayleigh line) skirts above the pressure-volume inflection point associated with the phase transformation and the conditions necessary for a further wave splitting due to the transformation are not achieved.

The phase transition is made apparent instead by a dramatic rarefaction shock wave which occurs during transformation reversal in the unloading wave structure. The steep drop ( $<10 \mathrm{~ns})$ attests to the very rapid kinetics associated with the reverse transition.

There does not appear to be a clear counterpart for this dynamic transition in the static literature. Aragonite is the stable reconstructed phase of calcium carbonate above approximately $0.3 \mathrm{GPa}^{12}$ Transformation from calcite to aragonite is resisted by a very substantial kinetic barrier and is further thwarted by the metastable displacive or neardisplacive calcite I-II and calcite II-III transitions in the 1-2 GPa range. The latter transitions exhibit similar characteristics under both shock and static conditions.

The new phase achieved under shock loading at 12-15 GPa (Figure 2) is not known. However, Kerley $^{13}$ has pointed out that equation-of-state properties of aragonite ${ }^{14}$ are fully consistent with the shock wave features contained in the high-pressure Hugoniot and shock profile data of calcite including that of Figure 2. The situation may again be that of achieving sufficient overpressure in the shock process to either drive or eliminate the transformation kinetics.

Wave profile measurements on aluminum nitride and calcium carbonate are illustrative of the clear signatures of phase transformation provided by the profile structure. The dominant signature for the former is in wave splitting of the shock compression profile - for the latter, in the release-wave rarefaction shock structure. 


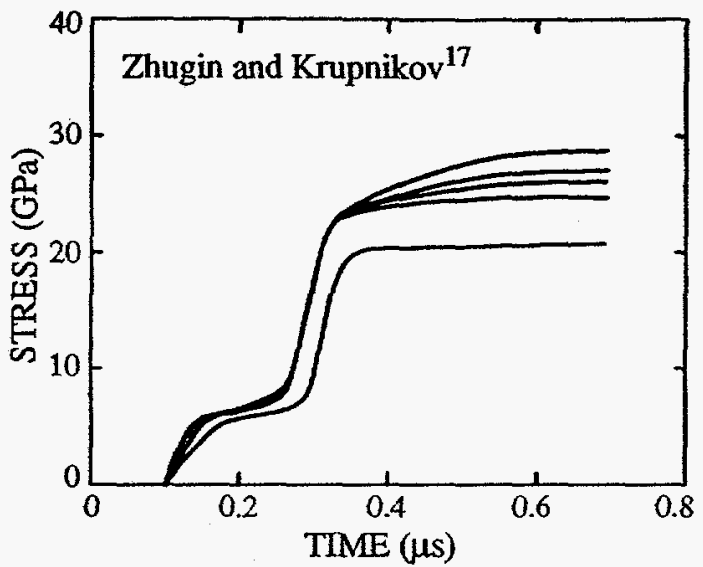

FIGURE 3. Manganin gauge stress profiles in quartzite. (Ref. 17)

Such obvious wave profile features associated with the massive tetrahedral-to-octahedral reconstructive phase transformation in quartz $\left(\mathrm{SiO}_{2}\right)$ and other silicate minerals have not been readily observed. Extensive earlier studies did not reveal a critical transition state on the quartz Hugoniot. Instead the data suggested a rather broad pressure range $(\sim 10-$ $40 \mathrm{GPa}$ ) over which the Hugoniot gradually transitioned through the mixed phase region - fully achieving the high density phase only after exceeding the mixed-phase pressure range. Several theories of phase transformation in the shock compression process were based on this observed "mixed-phase Hugoniot" of quartz and the quartz-like minerals. 15,16

A critical transition state and a definitive feature in the shock compression profile of quartz associated with the transition has now been observed. ${ }^{17}$ The reasons for this feature eluding the extensive earlier studies constitutes an interesting story in the development of experimental shock-wave technology.

Manganin gage profiles in Figure 3 of Zhugin and Krupnikov ${ }^{17}$ measured in quartz rock clearly indicate onset of high-pressure phase transformation at approximately $23 \mathrm{GPa}$. Profiles at increasing impact amplitudes identify the transition cusp. Further measurements with VISAR technology ${ }^{18}$ have provided independent evidence for the phase transformation structure in the compressive shock wave.

Because of the recent wave profile data which indicates a critical transition stress level for lattice instability in the quartz structure, both the interpretation of the earlier shock data and theories of the phase transformation in quartz should be revisited.

Recent static compression work of Hemley et al. ${ }^{19}$ should be noted in which a crystal-to-amorphous transition in quartz at approximately $20 \mathrm{GPa}$ was reported. The static transition in quartz has been explained in terms of free-energy/kinetics arguments ${ }^{20}$ in which the $\alpha$-quartz crystalline state is driven by elevated pressure to a high free energy state with respect to both the high density crystalline and amorphous state, but transformation is prohibited by transition kinetics. When the mechanical stability limits are achieved (spinodal decomposition) the material follows the easiest kinetic path to an amorphous high density state. The possibility of a solid-state transition to an amorphous phase was previousily suggested by DeCarli and Milton. ${ }^{21}$ Molecular dynamics simulations of the $\alpha$-quartz transition have been quite satisfactory in reproducing the expected transition pressure and volume strain. 22,23 Clearly this proposed transformation mechanism also warrants consideration for the shock transition in quartz, considering the remarkably close agreement between critical stress levels reported in the static work of Hemley et al., ${ }^{19}$ and in the dynamic studies of Zhugin and Krupnikov. ${ }^{17}$

Earlier interpretations of shock wave results in quartz and in other silicate minerals which led to a broad Hugoniot range (approximately 20-40 GPa) in which the shock velocity remained constant with increasing particle velocity are open to question. Wave structure above the $20 \mathrm{GPa}$ level is apparently subtle and requires high-fidelity wave profile diagnostics to resolve. Earlier shock-wave diagnostics would have easily missed this additional structure. The consequence was to report an unchanging shock velocity level (the transition shock velocity) and a corresponding increasing Hugoniot particle velocity level. Shock velocities would again begin to increase with particle velocity only after the $23 \mathrm{GPa}$ transition shock was overdriven.

As an aside, similar errors in Hugoniot data interpretation have been made on ground shock data in silicate geology. ${ }^{24}$ This experimental interpretation of the constant shock velocity region in quartz seems inherently more reasonable than earlier explanations based on equation-of-state or kinematic arguments. The recent data imply that the dynamic compression 
curve of $\alpha$-quartz would lie below the previously reported Hugoniot curve for quartz for pressures above the $23 \mathrm{GPa}$ level over the range of a separate transformation wave. It should be noted that Wackerle $^{25}$ was concerned about the possible existence of a third wave in this range but instrumentation at that time was not sufficient to resolve the details of this wave profile structure.

\section{FAILURE UNDER SHOCK COMPRESSION}

Failure in the brittle solid under the intense shear stresses developed in the shock compression process also plays an important role in determining the structure of wave profiles. Both plasticity and fracture are viable mechanisms for inelastic deformation under shock compression, although the relative importances of plasticity and fracture under the range of loading conditions experienced in the shock process have not yet been fully worked out.

The shock-wave precursor, or elastic precursor, is perhaps the most prominent wave profile feature thought to mirror the failure processes in the shock compression of solids. (Failure in this context refers to the abrupt softening in the axial modulus implied by the precursor wave structure.) Numerous studies have reflected on features of these precursor waves in brittle materials, and their relationships to the underlying deformation mechanisms. One feature which has received attention is the attenuation of precursor waves with propagation distance, and the attendant implications on the rate dependent nature of the inelastic failure processes. Sufficient investigations of this effect have been accomplished to provide an indication of the importance of precursor decay in brittle solids. Studies on hard rock materials including quartzite ${ }^{26}$ and dolomite 27 indicated modest elastic precursor decay, and Rosenberg et al. ${ }^{28}$ have reported elastic precursor decay in an aluminum oxide ceramic. Crystalline solids which undergo post-yield stress relaxation appear inclined to exhibit precursor decay. Examples include single crystal magnesium oxide 29 and forsterite. ${ }^{30}$ Boron carbide ceramic also exhibits precursor stress relaxation and precursor decay. ${ }^{31}$

On the other hand, the brittle solids which exhibit post-yield hardening apparently show less tendency toward precursor decay. Studies of Cagnoux and
Longy ${ }^{32}$ on aluminum oxide ceramics did not show elastic precursor decay, and profile measurements on dense limestone also indicated little or no precursor decay. ${ }^{33}$ Investigations of elastic precursor decay on a wider suite of ceramic materials ${ }^{34}$ also did not support extensive elastic precursor decay. For Hugoniot elastic limit data, and its dependence on strain rate extracted from the latter study and shown in Figure 4, only boron carbide shows some evidence for precursor decay. ${ }^{34}$ Also there are concerns that wave profile instrumentation with limited time resolution may falsely indicate more marked precursor decay than actually occurs.

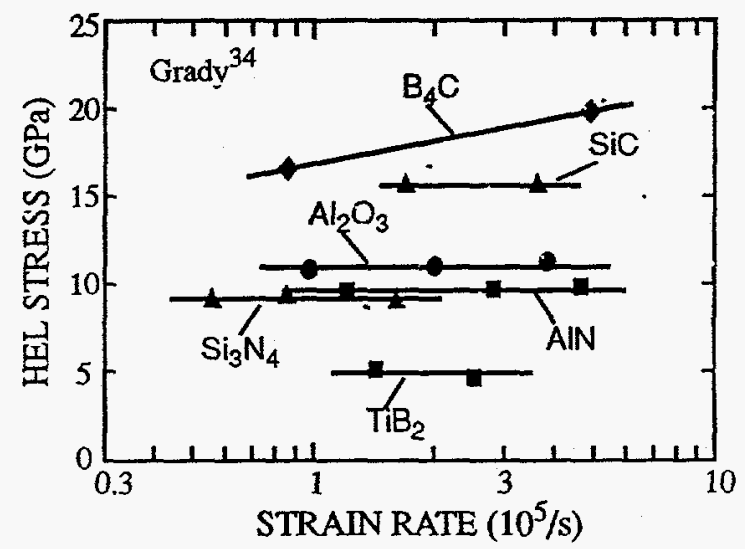

FIGURE 4. Hugoniot elastic limit stress versus strain rate for selected ceramics. (Ref. 34)

In general the shock-wave data suggest modest or negligible elastic precursor decay in most polycrystalline brittle solids. Consequently, very modest rate dependence in the processes governing failure and post-failure behavior in these materials would be implied.

The Hugoniot elastic limit strengths reported for a suite of high-strength ceramics ${ }^{35}$ corresponded to resolved shear stresses ranging from approximately $G / 25$ to $G / 40$ where $G$ is the shear modulus of the ceramic. Various authors (eg. Hirth and Lothe $\mathrm{e}^{36}$ ) have estimated the ultimate strength of a perfect closepacked lattice to lie between $G / 5$ and $G / 30$. Experimental studies on single crystal whiskers have achieved strengths as high as G/15. Dynamic strengths as high as $\mathrm{G} / 20$ have been obtained in shock wave studies on crystalline lithium fluoride. ${ }^{37}$ Dynamic failure (or yield) in lithium fluoride occurs by intracrystalline plasticity through dislocation motion and multiplication. Similar conclusions of 

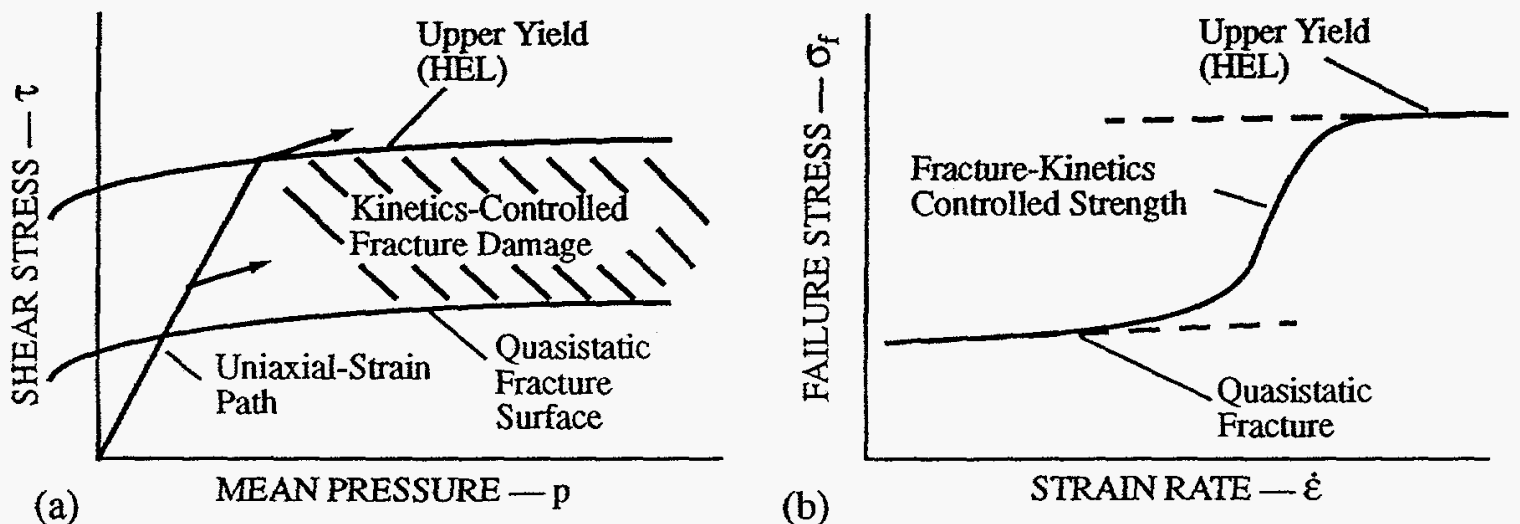

FIGURE 5. Dynamic failure in brittle solids based on fracture-kinetics and mechanism-transition model. (Ref. 27, 34)

intragranular plasticity mechanisms for shock-wavefailure and post-failure behavior have been reached in studies on polycrystalline aluminum oxide $\mathrm{e}^{38,39}$ and on dolomitic rock. ${ }^{27}$

Although a clear consensus has certainly not yet been reached, the evidence tends to suggest that failure at the $\mathrm{HEL}$ in the shock compression of many brittle solids is governed by the onset of crystal plasticity and that the modest inelastic deformations achieved in the shock process exhibit little strain rate dependence. The existence of porosity in the material is a possible complicating matter which is not considered here, but may when present dominate failure at the HEL. ${ }^{40}$

In any case the deformation behavior and weak strain-rate dependence associated with the yield of brittle solids at the HEL differs markedly from the strong strain-rate dependent brittle fracture processes characterizing failure in unconfined Hopkinson $\mathrm{bar}^{41}$ and dynamic spall experiments. ${ }^{42}$

In addition quasi-static triaxial and uniaxial compression studies on hard rock materials ${ }^{43}$ contribute to a picture of strain-rate dependence which is also not easily reconciled within more conventional models of failure and deformation.

To account for the range of effects observed in the dynamic and static failure of brittle solids a model of failure was introduced ${ }^{27}$ which accommodated features of both time-dependent fracture and rate dependent (or weakly rate dependent) plasticity. The essential features of the model are illustrated in Figure 5. On the left, within a plot of confining pressure versus shear stress, two uniquely different failure surfaces are identified. A path consistent with the uniaxial strain shock compression process is also shown, although other paths such as uniaxial stress might equally well be considered. The lower surface identifies a quasi-static fracture limit at which a critical flaw or system of flaws is activated by the stress load. Quasi-static or shock-wave loading below this surface is elastic. Dynamic or transient loads can exceed this quasi-static fracture surface because of time-dependence of the kinetics-controlled fracture damage process. Because of the very high strain rates associated with the shock compression process, stress levels consistent with the upper yield can be achieved with negligible fracture damage. When the upper yield is reached in the shock compression process (the HEL) a transition in shear failure mechanisms is achieved.

The dependence of the strength of the material on strain rate is illustrated on the right of Figure 5. Such a plot would represent, for example, strength data under uniaxial stress (Hopkinson bar), or uniaxial strain (shock wave), loading. Occasionally such data are mixed, making reasonable corrections for differences in loading paths. At lower strain rates, strength is governed by onset of fracture with a relatively modest strain rate dependence due, in certain brittle solids, to thermally-activated subcritical crack growth. ${ }^{41}$ This strength level is identified by the lower quasi-static fracture curve in the right of Figure 5.

At a characteristic transition strain rate a regime is entered in which athermal, inertia-dominated fracture damage kinetics lead to a rapidly increasing failure 

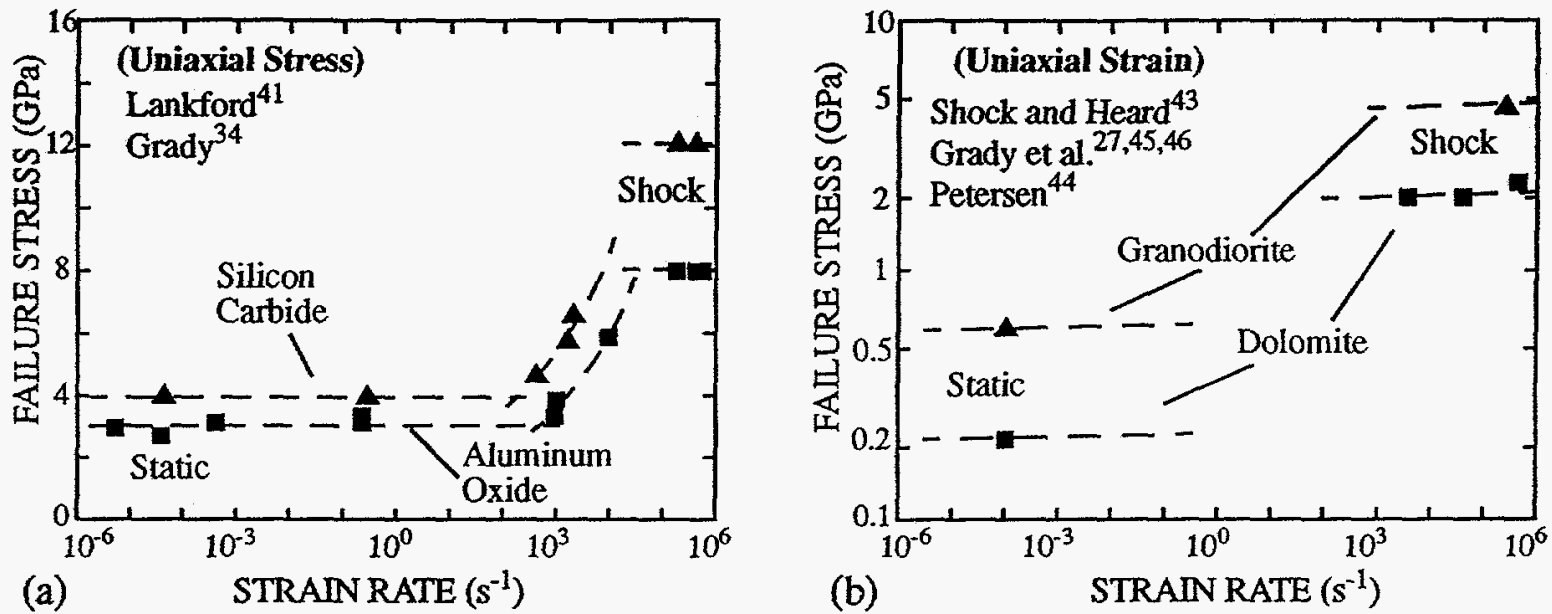

FIGURE 6. Dynamic failure properties of selected brittle solids under quasistatic and dynamic compressive loading.

stress with strain rate. At the highest strain rates the delay due to damage kinetics precludes failure by brittle fracture and an alternative failure mechanism is activated - reflected by the upper yield surface (the HEL) in Figure 5. In this regime a more modest strain rate dependence associated with this upper yield surface is consistent with the observed HEL behavior in brittle solids (Figure 5). These trends in the behavior of dynamic failure could be interpreted as a strain-rate dependent brittle-to-ductile transition in the failure mechanism.

Data supporting fracture-damage kinetics and a mechanism transition in the dynamic failure of brittle solids are shown in Figure 6. On the left quasi-static and Hopkinson bar data of Lankford ${ }^{41}$ are combined with shock wave data (Figure 6) for aluminum oxide and silicon carbide. ${ }^{34}$ These data clearly indicate an entry strain rate to a kinetics-controlled failure strength regime and further suggest transition to an alternative rate-insensitive failure mechanism at the higher, shock-wave strain rates. Worthy of observation is that the transition to a rapidly increasing strength regime occurs for aluminum oxide at a higher strain rate than for silicon carbide. The difference would imply that fracture damage kinetics is somewhat faster in aluminum oxide. The controversial nature of Hopkinson bar data in the high-strain-rate regime, and the mixing of uniaxial stress and strain data in this plot, make conclusions from these data tentative.

The plot on the right includes both static ${ }^{43}$ and shock $^{27,44}$ uniaxial-strain strength-at-failure data for
Blair dolomite and Climax Stock granodiorite. For the dolomite, impact experiments involving shock wave, ${ }^{27}$ ramp wave ${ }^{45}$ and shock dwell ${ }^{46}$ techniques failed to reveal the rate dependent strength intplied by the markedly lower quasi-static strength value. Ramp wave and shock wave studies on aluminum oxide ${ }^{32}$ similarly failed to identify significant strain-ratedependent strength in the high strain-rate region accessed by impact techniques. The above model would predict a rapidly rising damage-kinetics controlled region joining the high-strain-rate and quasi-static data.

An alternative view of the effects of damage kinetics on the failure process in brittle solids is provided by considering the shock-wave (or Heaviside) compressive loading to states below the HEL, but above the quasi-static fracture surface. The initial response is expected to be elastic. Shear failure through a fracture process and controlled by the brittle fracture damage kinetics is predicted by the model to occur after sufficient time at stress, however. ${ }^{27}$ Characteristic delay times of microseconds to tens of microseconds have been predicted for silicon carbide and aluminum oxide based on the data of Lankford ${ }^{41}$ and a damage kinetics model ${ }^{34}$

An alternative explanation for delayed fracture following an initial elastic shock wave has been proposed by Nikolaevskii. ${ }^{47}$ Failure through fracture is predicted to proceed through a following failure wave at a characteristic speed governed by a limiting fracture velocity.

The evidence for delayed shear failure and failure 


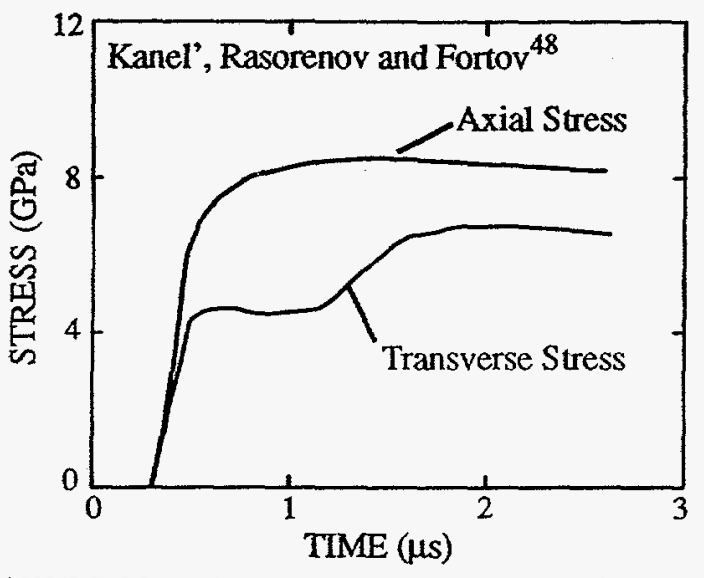

FIGURE 7. Manganin gauge profiles in glass measuring axial and transverse stress in a compressive shock wave. (Ref. 48)

waves in the shock compression of brittle solids is mounting. Experiments of Kanel' et al. ${ }^{48}$ on glass show evidence of wave refiections from propagating damage fronts, while measurements of lateral and longitudinal stress (Figure 7), indicate a delayed collapse of the stress difference associated with the failure process, provide quite convincing proof of the existence of failure waves. Further work summarized in Brar et al. ${ }^{49}$ confirm the results of Kanel' et al. ${ }^{48}$ in glass, note the possible occurrence of dilatancy in the damage wave implied by the stress profile data, and describe spall studies demonstrating loss of tensile strength behind the damage front. Dandekar and Mole $^{50}$ have noted a tendency toward a constant failure wave velocity in glass, at least for a fixed impact amplitude. Photographic studies of Brannon et al..$^{51}$ on crystalline quartz, Barker et al. ${ }^{52}$ on brittle plastics, and Boume et al. ${ }^{53}$, Senf and Strausburger ${ }^{54}$ and Bless et al. ${ }^{55}$ on glass all provide visual evidence for failure waves in brittle solids.

\section{VISCOSITY OF SOLIDS IN SHOCK COMPRESSION}

A behavior peculiar to nonlinear waves is the development of shock profiles which propagate unchanged in form. Such waves of steady profile structure (steady waves) rise from the wave trough to the wave crest over a finite time which is determined by both material properties and the amplitude of the wave. The amplitude dependence of the risetime has been shown to exhibit a more or less common trend over a range of metallic materials. ${ }^{56,57}$ Recent shock profile measurements on soda-lime glass shown in Figure 8 have been found to exhibit the same, previously observed, behavior. In Figure 9 a plot of the stress jump through the wave plotted against the maximum strain rate in the wave shows the fourth power behavior observed previously. This behavior has been used as a "law of solid viscosity" in development of visco-plastic models which reasonably reproduce the observed profile shapes in shock propagation applications $57,58,59$.

Although the mechanics through which rate dependence or viscosity leads to the evolution of steady waves are reasonably well known, the underlying molecular or microstructure mechanisms responsible for the constitutive behavior remains poorly understood. Race dependent plasticity with attendant inelastic dislocation movement governed by the thermally-activated penetration of energy barriers represents a nonviscous mechanism for establishing finite risetime steady waves. Of interest in this discussion is the range of viscosity-based mechanism which may be active within shock waves in solid materials.

Viscosity can be defined as the transport of momentum or kinetic energy along a velocity gradient. In a kinetic model of the viscosity process, a quantum of momenta separates from the local flow and is transported along the velocity gradient a characteristic length before reimpingement. It adds to, or subtracts from, the momentum at the new point. In the steady shock-wave process, viscosity can be viewed as the diffusion of momentum along the axis of wave propagation. Viscosity is commonly considered as a material constant which provides a proportionality between a viscous stress component and the velocity gradient or strain rate. More complex viscous constitutive behavior is observed in the rheology of solids.

The magnitude of a viscosity coefficient $\eta$ expected in a steady shock wave process can be estimated through the material parameters which establish the upward concavity of the Hugoniot curve, and the geometry of the Rayleigh line corresponding to a specific steady shock event. Such an estimation is based on the assumption of a decomposition of the dynamic stress into an equilibrium elastic and a viscous component. The first pressure derivative of 


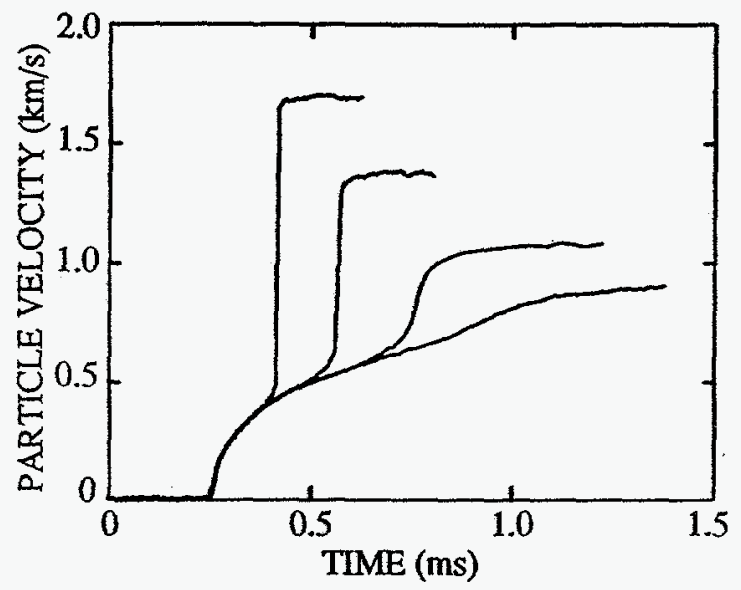

FIGURE 8. Wave profiles in soda-lime glass illustrating shackformation and steady-rise-time features.

the bulk modulus $K$ or, more common in shock wave analysis, the parameter $S$ in a linear-shock-velocityversus-particle-velocity relation, provides a dimensionless measure of the concavity of the Hugoniot and the rate at which a finite-amplitude compression wave will steepen or "shock up" with propagation distance. On the other hand the ratio $\eta / \sigma \tau$, where $\sigma$ and $\tau$ are the jump in stress amplitude and the risetime of the wave, respectively, provides an opposing dimensionless force determining the rate of wave spreading. A steady wave is achieved when a balance, $S \sim \eta / \sigma \tau$, of the opposing forces is achieved. An only slightly more rigorous analysis, based on the geometry of the Hugoniot and the Rayleigh line, leads to the following expression for the viscosity in a steady-shock wave,

$$
\eta \cong \frac{1}{4} S \sigma \tau \text {. }
$$

Brittle solids are observed to exhibit measurable rise times in the deformation shock wave provided the shock amplitude of the order of or less than a few times the magnitude of the Hugoniot elastic limit. ${ }^{60}$ Representative values would be a 10 GPa shock jump and a $0.1 \mu$ s rise time. With a value for $S$ of unity, Equation 1 provides $\eta \sim 250$ Pa.s for an order of magnitude estimate of the expected viscosity. If a constant viscosity coefficient governs the steady shock-wave formation, Equation 1 implies that the risetime of the steady wave decreases as the inverse first power of the stress amplitude. Wave profile

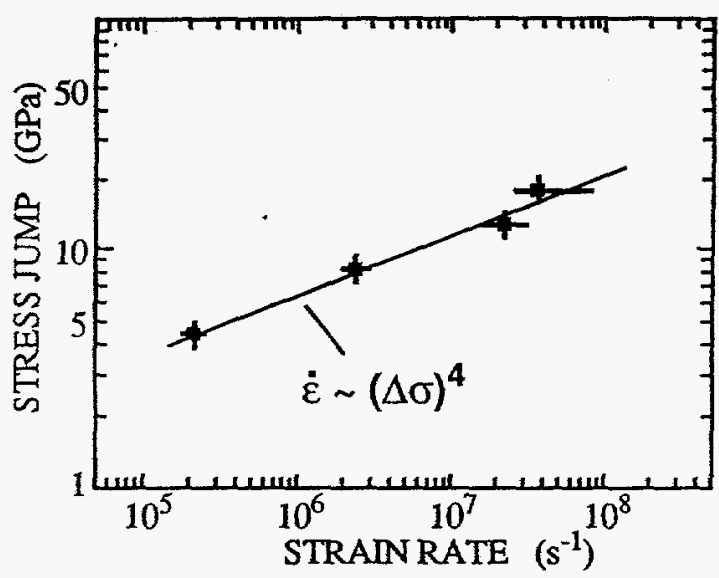

FICURE 9. Rise time versus strain rate data for shock wave profiles in Figure 8.

measurements, of which the data in Figure 8 are representative, reveal a much more rapid decrease in risetime, suggesting a decreasing viscosity with stress amplitude.

If viscous processes are responsible for the finite thickness of steady shock waves in solids, then the underlying physical mechanisms of solid state viscosity are the basis of a fuller understanding of shock processes in solids. Gilman ${ }^{61}$ has explored sources of viscous drag stress in metals based on a kinetics of momentum diffusion of atomistic processes active in the shock wave front. Contributions from the electron and phonon gas, and atomic interactions were considered. Estimates of mass fluxes and transport cross-sections were found to lead to negligible contributions to viscous drag for most of the processes examined. Only dislocation drag effects were found to contribute significantly to shock viscosity.

If the mechanisms within the shock front are widened to include microstructural as well as molecular processes, additional mechanisms contributing to viscosity are possible, and are perhaps more relevant to the brittle solid. Although the kinetic diffusion of mass provides a principal mechanism for viscosity in fluids, mass diffusion seems a less likely viscosity mechanism in the shock compression of solids. Evidence supporting some degree of mass diffusion in the shock front will be mentioned later, however, possibly suggesting some viscous contribution from this process. 
A microstructural analog to phonon viscosity (the diffusion of momentum through acoustic energy) seems relevant to the brittle solid. Defects on all scales (dislocation, vacancies, porosity, granularity, lamination, etc.) provide sites for wave scattering. Also unstable failure on the microscale due to fracture or adiabatic slip can lead to acoustic emission. Both acoustic scattering and acoustic emission provide a mechanism for diffusion of momentum along the axis of the propagating wave. By such processes coherent energy within the wave front can be transferred to incoherent energy behind the wave. That this "dissipated energy" may still reside as random acoustic energy at the microstructural scale rather than thermal kinetic energy at the molecular scale is not important to features of the wave front profile. The consequences in terms of the viscous character of wave propagation are the same.

In fluids a kinetic theory of the random transport of momentum has had a reasonable measure of success in providing insight into a fuller understanding of viscosity in those materials. A relation for the viscosity coefficient based on kinetic theory can be expressed in various ways. One form that was developed to examine an application to wave structure in solids ${ }^{62}$ is,

$$
\eta \equiv \frac{2}{3} N E_{k} \tau_{c} .
$$

In Equation $2 N$ is the number of momentum transporting elements per unit volume, $E_{k}$ is the kinetic energy per element and $\tau_{c}$ is the characteristic collision time. Equation 2 was used by Passman et al. ${ }^{62}$ to examine wave profile structure in the spall of brittle solids. Momentum transporting elements were assumed to be the tips of propagating microcracks and viscosity in the failure process was established through Equation 2.

An obvious example, in which elastic scattering plays an important role, is the propagation of shock waves in heterogeneous materials. Notable is the extensive study by Barker $^{63}$ on laminates of dissimilar materials in which he. clearly notes the viscous nature of the wave propagation process.

It is of interest to estimate the viscosity within the shock wave propagating through such a material with the kinetic relation in Equation 2. As a representative example consider a laminate of alternating $1-\mathrm{mm}$ thickness boron carbide and aluminum oxide plates. From the analysis of Barker ${ }^{63}$ one can estimate $E_{k} \sim(1-F)\left(\rho p^{2} / 2 Z\right)$ and $\tau_{c}-2 h / U_{s} \ln (1 / F)$ where $F=4 Z_{1} Z_{2} /\left(Z_{1}+Z_{2}\right)^{2}$. In these relations $Z$ is a mean laminate impedance, while $Z_{1}$ and $Z_{2}$ are individual component impedances, and $h$ is the plate thickness. For a $10 \mathrm{GPa}$ shock wave Equation 2 yields approximately $\eta \sim 20 \mathrm{~Pa} \cdot \mathrm{s}$.

One expects a steady shock in such a laminate to span at least several cell dimensions and perhaps more. (Resonances achieved in the post-shock structure in evenly spaced laminates are ignored here.) This would require from Equation 1 a viscosity of about $500 \mathrm{~Pa} \cdot \mathrm{s}$ or greater - a marked discrepancy with the kinetic-theory-based prediction. The difference suggests an inadequacy in the logic leading to one or the other predictions.

There are several possible considerations which may introduce difficulties in a simple model which superposes an equilibrium elastic stress and a viscous stress to achieve steady shock waves, and in a kinetic theory of momentum diffusion to estimate viscous coefficients.

First, within a heterogeneous solid (all solids are heterogeneous to some degree), during the passage of a steady shock wave, it is highly unlikely that an equilibrium elastic state will be achieved within the large velocity gradient portion of the wave. A Maxwell viscoelastic model, as was suggested by Barker $^{63}$ for a laminated solid, in which a viscous transition from an instantaneous to a equilibrium elastic state is allowed, is probably a much better representation of the underlying elasticity in the physics of steady shocks in heterogeneous matter.

Second, the elements of momentum diffusion responsible for viscous dissipation in steady shock propagation are fundamentally different from those considered in the more common kinetic theories of fluids. In the latter those elements, whether particle like or phonon like, are a consequence of the underlying thermal motion of the body. Wave motion and velocity gradients introduced into this body would recognize this motion as viscosity but are not responsible for them. In the steady shock waves of interest here, the elements of momentum diffusion (scattered elastic waves, acoustic emission, microfractures, ...) are, in contrast, stimulated, or induced, by the wave. In some cases the mean free 
paths of these elements may exceed the characteristic wave length of the steady-wave profile. There may be a role for kinetic theories of viscosity in the steadywave shock process, but a direct adoption of traditional fluid theories without due reflection would be suspect.

Finally, an observation on mass diffusion in the steady shock wave, within the context of shock viscosity, is worthy of note. In the shock process in solids, even for relatively intense shocks, material elements (atoms and molecules) are expected to remain at or close to their original lattice points. Dremin and Breusov ${ }^{64}$, proposed a model allowing some material mixing through the inelastic shearing of neighboring planes, but even here relative motions were not expected to be large. Alekseevskii et al., 65 however, have experimentally investigated shock wave propagation in several metals in which radioisotopes were placed on an interface which infercepted the passing shock waves. In post-test analysis it was found that the radioactive atoms were transported a considerable distance (a number of millimeters) into the material - the quantity of mass transfer dependent on the shock intensity and the strength of atomic bonds in the substance. A clear explanation for this anomalous diffusion effect was not provided, although the relatively low transport energy of interstitial atoms in the shock front was suggested. Gluzman and Psakh' $e^{66}$ suggested that in such an effect, a low density of solitons propagating in the shock may be the carriers in the anomalous mass transport process. The present author suspects that selected atoms, whose thermal kinetic energy is favorably disposed upon arrival of the shock wave, can "ride-the-wave" for some significant distance before dropping behind the wave due to perturbations from lattice imperfections. In any case these data suggest that momentum diffusion due to mass transport in the solid state is apparently possible. Again it is a situation where the diffusing elements are stimulated by the passing shock wave.

\section{CLOSURE}

Shock wave studies on brittle solids initiated some three decades ago with the research on engineering ceramics [e.g. Gust and Royce ${ }^{67}$ ] and on rocks and minerals [e.g. Ahrens and Gregson. ${ }^{68}$ ] have provided the seminal efforts in an extensive body of literature dedicated to discovering the shock properties of these materials. The nonlinear features of brittle materials, as reflected in the richness of measured shock wave profiles, attests to the complexities of physical and mechanical processes active in the shock wave event. Unraveling the relative roles of these processes has been difficult and only recently have some of the more important mechanisms come to light. The shock-wave and high-strain-rate properties of brittle solids can be expected to challenge the experimenter and theorist for some time to come.

\section{REFERENCES}

1. Duvall, G. E. and Graham, R. A., Rev. Modern Phys., 49, 523579 (1977).

2. Shono, Y., Shock Waves in Condensed Matter - 1987, Elsevier Science Publ., 1988, pp. 19-26.

3. Kondo, K. A., Sawaoka, A., Sato, K., Ancio, M., Shock Waves in Condensed Matter - 1981, AIP Press, 1982, pp. 303-305.

4. Vollstadt, H. E., Ito, E., Akaishi, M., Akimoto, S., Fukunaga, O., Proc. Japan Acad., 66 Ser.B, 7-9 (1990).

5. Jones, O. E. and Graham, R. A., "Shear Strength Effects on Phase Transition Pressures Determined From ShockCompression Experiments", presented at the Symposium on Accurate Characterization of the High-Pressure Environment, Gaithersburg, Md., October 14-18, 1968.

6. Kipp, M. E. and Grady, D. E., DYMAT 94 International Conference on Mechanical and Physical Behaviour of Materials under Dynamic Loading, les Editions de Physique, 1994, pp. 249-256.

7. Nakamura, A. and Mashimo, T., High-Pressure Science and Technology - 1994, AIP Press, 1994, pp. 303-305.

8. Dandekar, D. P., Abbate, A., Frankel, J., J. Appl. Phys., 76, 4077-4085 (1994).

9. Xia, Q., Xia, H., Ruoff, A. L., High-Pressure Science and Technology - 1994, AIP Press, 1994, pp. 307-310.

10. Grady, D. E., Shock Waves in Condensed Matter - 1985, Plenum Publ., 1986, pp. 589-593.

11. Aidun, J. B. and Gupta, Y. M., J. Geophys. Res., 100, 19551980 (1995).

12. Jamieson, J. C., J. Chem. Phys., 21, 1385-1390 (1953).

13. Kerley, G. I., Shock Waves in Condensed Matter - 1989, AIP Press, pp. 613-616.

- 14. Vizgirda, J. and Ahrens, T. J., J. Geophys. Res., 87, 4747-4758 (1982).

15. Grady, D. E., J. Geophys. Res., 82, 913-924 (1980).

16. Podurets, A. M. and Trunun, R. F., Soviet Physics - Doklady, 15, 1117-1118 (1971).

17.Zhugin, Yu. N. and Krupnikov K. K., Transactions of the XI International IUPAP-IUTAM Symposium on Non-Linear Acoustics, Part II, Novosibirsk, Russia, 1987, pp. 196-200. 
18. Grady, D. E. and Zhugin, Yu. N., (Abstract only) Bull. Am. Phys. Soc., 39, 410-411 (1984) (also Grady SAND Rept). also Grady, D. E., Sandia National Laboratories Technical Rept. SAND94-3266, February (1995).

19. Hemley, R. J., Jephoaat, A. P., Mao, H. K., Ming. M. H., Manghnani, M. H., Nalure, 334, 254-258 (1988).

20. Sikka, S. K., Recent Trends in High Pressure Physics, Oxford and IBH Publ., 1992, pp. 254-258.

21. Decari, P. S. and Milton, D. J., Science, 147, 144-145 (1965).

22. Tse, J. S. and Klug, D. D., Recent Trends in High Pressure Physics, Oxford and IBH Publ., 1992, pp. 274-279.

23. Chaplot, S. L. and Sikka, S. K., Recent Trends in High Pressure Physics, Oxford and IBH Publ, 1992, pp. 259-261.

24. Grady, D. E., Sandia National Laboratories Technical Rept. SAND91-2000, November (1991).

25. Wackerie, J,, J. Appl. Phys., 33, $922-937$ (1962).

26. Ahrens, T. J. and Duvall, G. E., J. Geophys. Res., 71., 4349-4360 (1966).

27. Grady, D. E., Hollenbach, R. E., Schuler, K. W., Callender, J. F., J. Geophys. Res., 82, 1325-1333 (1977).

28. Rosenberg, Z., Brar, N. S.; Bless, S. J.,DYMAT 88 International Conference on Mechaniçal and Physical Behaviour of Materials under Dynamic Loading, les Editions de Physique, 1988, pp. 707-711.

29. Grady, D. E., High Pressure Research: Applications in Geophysics, Academic Press, 1977, pp. 389-438.

30. Fumish, M. D., Grady, D. E., Brown, J. M., Shock Waves in Condensed Matter - 1985, Plenum Press, 1986, pp. 595-600.

31. Grady, D. E., DYMAT 94 International Conference on Mechanical and Physical Behaviour of Materials under Dynamic Loading, les Editions de Physique, 1994, pp. 385-391.

32. Cagnoux, J. and Longy, F., Shock Waves in Condensed Matter - 1987, Elsevier Publ., 1988, pp. 293-296.

33. Grady, D. E., Sandia National Laboratories Technical Rept. SAND83-0370, March(1983).

34. Grady, D. E., Constitutive Modeling: Theory and Applications, CIMNE, Barcelona, 1995 (to be published).

35. Grady, D. E., Shack Waves in Condensed Matter - 1989, AIP Press, 1990, pp. 377-380.

36. Hirth, J. P. and Lothe. J., Theory of Dislocations, McGraw Hill, 1968.

37. Gupta, Y. M., Bull. Am. Phys. Soc., 2, 50 (1975).

38. Munson, D. E. and Lawrence, R. J., J. Appl. Phys., 50, 6272$6282(1979)$

39. Longy, F, and Cagnoux, J., J.Amer. Ceramic. Soc., 72, 971-979 (1989).

40. Rosenberg, Z., Shock and Impact on Structures, Computational Mechanics Pubt, Boston, 1994, pp. 73-105.

41. Lankford, J., J. Amer. Ceramic. Soc., 64, C33-C34 (1981).

42. Grady, D. E. and Kipp, M. E., Structural Failure, John Wiley and Sons, 1989 , pp. $1-40$.

43. Shock, R. N. and Heard, H. C., J. Geophysics Res., 79, 1662 1666 (1974).
44. Petersen, C.F., Ph. D. Dissertation, 92 pp., Stanford University, Stanford Califomia (1969).

45. Grady, D. E., Geophys. Res. Letts., 1, 263-266 (1977).

46. Grady, D. E., Sandia National Laboratories Technical Rept. SAND83-0898, September (1983).

47. Nickolaevskii, V. N., Int. J. Engng. Sci., 19, 41-56.

48. Kanel', G. I., Rasonenov, S. V., Fortov, V. E., Shock Waves in Condensed Matter - 1991, Elsevier Science Publ, 1992. pp. $451-454$.

49. Brar, N. S., Bless, S. J., Rosenberg, Appl. Phys. Letts., 59, 33963398 (1991).

50. Dandekar, D. D. and Mole, P., Metallurgical and Materials Applications of Shock-Wave and High-Strain-Rate Phenomena, (in Press), 1995.

51. Brannon, P. J., Konrad, C., Morris, R. W., Jones, E. D., Asay, J. R., J. Appl. Phys., 54, 6374-6381.

52. Barker, D. B., Foumey, W. L., Dally, J. W., Photoelastic Investigations of Fragmentation Mechanisms, University of Maryland Technical Rept., March (1978).

53. Boume, N. K., Rosenberg, Z., Mebar, Y., Obara, T., Field, J.E., DYMAT 94 International Corference on Mechanical and Physical Behaviour of Materiais under Dynamic Loading, les Editions de Physique, 1994, pp. 635-640.

54. Senf, H., Strausburger, E., Rothenhausier, DYMAT 94 International Conference on Mechanical and Physical Behaviour of Materials under Dynamic Loading, les Editions de Physique, 1994, pp. 741-746.

55. Bless, S. J., Brar, N. S., Kanel, G., Rosenberg, Z., J. Am. Ceram. Soc., 75, 1002-1004 (1992).

56. Grady, D. E., Appl. Phys. Lett., 38, 825-826 (1981).

57. Swegle, J. W. and Grady, D. E., J. Appl.Phys., 58, 692-701 (1985).

58. Rubin, M. B., Shock Waves in Condensed Matter - 1989, Elsevier Publ., 1990, pp. 321-328.

59. Johnson, J. N., J. Appl. Phys., 72, 797-799 (1992)

60. Kipp, M. E. and Grady, D. E., Sandia National Laboratories Technical Rept. SAND89-1461, July(1989).

61. Gilman, J. J., J. Appl. Phys., 50, 4059-4064 (1979).

62. Passman, S. L., Grady, D. E., Rundle. J. B., J. Appl. Phys., 51, 4070-4075 (1980).

63. Barker, L. M., J. Composite Materials, 5, 140-162 (1971).

64. Dremin, A. N. and Breusov, G. A., Russian Chem. Rev., 37, 396-402 (1964).

65. Alekseevskii, V. P., et. al., Sov. Powder Metall. Metal Ceram., 10, 809-813 (1989).

66. Gluzman, S. L. and Psakh'e, S. G., Sov. Tech.Phys. Lett., 15, 764-768 (1989)

67. Gust, W. H. and Royce, E. B., J. Appl. Phys., 42, 276-294 (1971).

68. Ahrens, T. J. an Gregson, V. G., J. Geophys. Res., 69, 48394874 (1964). 